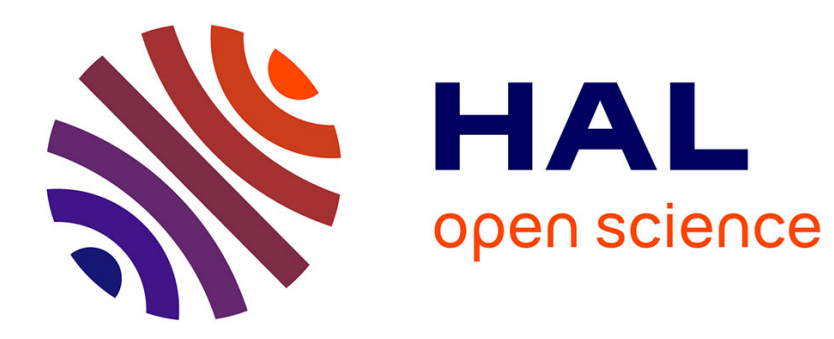

\title{
A New Methodology for Observer Design and Implementation
}

\author{
Ramine Nikoukhah
}

\section{To cite this version:}

Ramine Nikoukhah. A New Methodology for Observer Design and Implementation. [Research Report] RR-2677, INRIA. 1995. inria-00074014

\section{HAL Id: inria-00074014 \\ https://hal.inria.fr/inria-00074014}

Submitted on 24 May 2006

HAL is a multi-disciplinary open access archive for the deposit and dissemination of scientific research documents, whether they are published or not. The documents may come from teaching and research institutions in France or abroad, or from public or private research centers.
L'archive ouverte pluridisciplinaire HAL, est destinée au dépôt et à la diffusion de documents scientifiques de niveau recherche, publiés ou non, émanant des établissements d'enseignement et de recherche français ou étrangers, des laboratoires publics ou privés. 
INSTITUT NATIONAL DE RECHERCHE EN INFORMATIQUE ET EN AUTOMATIQUE

\section{A new methodology for observer design and implementation}

Ramine Nikoukhah

\section{N 2677}

October 1995

PROGRAMME 5 



\title{
RIN RIA
}

\section{A new methodology for observer design and implementation}

\author{
Ramine Nikoukhah \\ Programme 5 - Traitement du signal, automatique et productique \\ Projet Meta2
}

Rapport de recherche $\mathrm{n}^{\circ} 2677$ - October 1995 - 25 pages

\begin{abstract}
Observers are usually formulated as explicit systems of differential equations and implemented using standard ODE solvers. In this paper, we show that there can be advantages in formulating the observer as a DAE (Differential-Algebraic Equation). A canonical DAE observer is proposed and it is shown that it can be implemented using standard DAE solvers. The DAE implementation of the observer can be considered regardless of the design philosophy used (extended linearization, Lyapunov based, Lie algebraic, ...). We present a simple design strategy for this canonical DAE observer based on the extended-linearization method.
\end{abstract}




\section{Une nouvelle méthode de conception et d'implémentation d'observateur}

Résumé : Dans ce rapport, on montre qu'il peut être avantageux de formuler le problème de l'observateur sous la forme d'un système implicite particulier qui peut être résolu par les solveurs existants. Cette formulation implicite peut être considérée indépendamment de la technique de conception utilisée (linéarisation étendue, Lyapunov, nonlinéaire,...). On présente une stratégie simple pour la construction d'un observateur implicite dans l'optique de la linéarisation étendue. 


\section{Introduction}

We consider the problem of observer design for multivariable nonlinear systems with $m$ inputs and $p$ outputs described by

$$
\begin{aligned}
\dot{x} & =f(x, u) \\
y & =h(x) .
\end{aligned}
$$

The state $x$ is assumed to belong to an open set $\mathcal{X} \subset \mathbb{R}^{n}$ and input $u$ to an open set $\mathcal{U} \subset \mathbb{R}^{m}$. We assume that $f$ and $h$ are $\mathcal{C}^{2}$ vector fields on $\mathbb{R}^{n}$ and $\mathbb{R}^{p}$, and that the $p$ measurements in (1.2) are independent.

The problem of observer design consists of finding a scheme for reconstructing the state $x$ based on past and present (known and/or measured) values of the input $u$ and the output $y$. This problem has been extensively studied in the past, both for linear and nonlinear systems. There are essentially two approaches to the problem of state reconstruction for nonlinear systems. In the first approach, which is a natural extension of linear observers and is very commonly adopted (for example see the techniques presented in the comparative study of [15]), the objective is to construct a vector field $k$ such that the solution $\hat{x}(t)$ of the observer

$$
\dot{\hat{x}}=k(\hat{x}, u, y)
$$

converges to the true value of the state $x$ (in some design methods, $k$ can also depend on time and/or the derivatives of $u$ ). The advantage of this approach is that (1.3) is an explicit system and can be solved using standard ODE (Ordinary Differential Equation) solvers. The other approach to observer design is to work directly with system equations (1.1)-(1.2), either formulating the estimation problem as a nonlinear algebraic system of equations which must be solved periodically using for example Newton's method (see for example [12]), or formulating it as an optimization problem over some sliding finite horizon which is again solved periodically [11]. This second approach has many advantages because it is not constrained by the "artificial" requirement that the solution be expressed in the form (1.3); the drawback is that it requires development of specific code for its implementation.

In this report, we present an alternative to these two approaches. In particular, we show that there can be advantages in formulating the observer as an implicit (descriptor) system which can then be solved using a DAE (Differential-Algebraic Equation) solver. Even though this method is closer in spirit to the second approach described above, because DAE solvers compute the solution of algebraic equations by Newton iterations at every time step, it does not require any custom code development.

Allowing the observer to be an implicit system provides an additional degree of freedom which is useful regardless of the observer design method used (extended linearization, Lyapunov based, Lie Algebraic,...). We shall focus our attention on extended-linearization method and propose a simple strategy for the design and implementation of a DAE observer based on this method.

In Section 2, we propose a canonical DAE observer and study some of its properties. Extended-linearization approach is used in Section 3 for developing a method for choosing

$\mathrm{RR} \mathbf{n}^{\circ} 2677$ 
the parameters of this DAE observer to meet needed performance and stability criteria. Section 4 is devoted to implementation issues. An example is presented in Section 5.

\section{Descriptor formulation of the observer}

System (1.1)-(1.2) is a DAE in $x$ ( $u$ and $y$ are supposed to be known). This over-determined ( $n$ unknowns, $n+p$ equations) DAE describes all the constraints (information) that we have for constructing $\hat{x}$. To make this DAE integrable, we can do relaxation by introducing a p-dimensional vector $\lambda(t)$ into this DAE. One way to introduce $\lambda$ is as follows:

$$
\begin{aligned}
\dot{\hat{x}} & =f(\hat{x}, u)+g(\lambda) \\
0 & =y-h(\hat{x})+\lambda
\end{aligned}
$$

This DAE is very special because we can easily solve for $\lambda$ in (2.2) and substitute the result in (2.1) obtaining an ODE; the result is an observer of the form (1.3). This construction is used (even though it is not presented this way) in most full-order observer design methods.

So, we see that introducing $\lambda$ as in (2.1)-(2.2) leads to the usual explicit formulation of the observer. It is surprising to see that relaxation is introduced in (1.2), specially if the measurement noise is small, since this equation gives directly the value of a projection of $y$ and constructing an $\hat{x}$ that satisfies this equation is in general not very difficult. So let us not introduce $\lambda$ in (1.2) and consider the DAE obtained from (2.1) and

$$
0=y-h(\hat{x}) .
$$

It turns out this formulation is closely related to reduced-order observers. The index of this $\mathrm{DAE}$ is at least 2 and can be arbitrarily large (index of a DAE is the number of times we need to differentiate the algebraic equations to obtain an ODE, see [5]). This can make the solution very sensitive to noise (see Example 2.3); this of course is a well known problem of reduced-order observers [6]. In fact, in general, this DAE cannot be solved by existing DAE solvers because, even though recently powerful DAE solvers have been developed for index 2 and index 3 systems, the only case where DAE solvers do not require that the system have any special structure and work as reliably as ODE solvers do, is the index 1 case. In fact, what really makes the DAE (2.1)-(2.2) tractable is that it has index 1 -differentiating (2.2) once yields an ODE in $\hat{x}$ and $\lambda$ - and not its special form.

\subsection{Canonical DAE observer}

In formulating the DAE observer, we like to introduce the relaxation variable $\lambda$ in such a way that the index of the resulting DAE does not exceed 1 without introducing $\lambda$ into (1.2).

Lemma 2.1 The following DAE has index 1:

$$
\begin{aligned}
\dot{\hat{x}} & =f(\hat{x}, u)+h_{x}(\hat{x})^{T} \dot{\lambda}+v(u, \hat{x}, \lambda, t) \\
0 & =y-h(\hat{x})
\end{aligned}
$$


where $f$ and $h$ are defined as in (1.1)-(1.2), $h_{x}$ denotes the Jacobian matrix $\frac{\partial h}{\partial x}$ and $v$ is any function which may depend on $u, \hat{x}, \lambda$ and time.

Note that allowing $v$ to depend on $y$ does not increase the generality of the formulation because $y=h(\hat{x})$.

Proof: By differentiating (2.5), we obtain:

$$
h_{x}(\hat{x}) \dot{\hat{x}}=\dot{y}
$$

which combined with (2.10) yields the DAE

$$
\left(\begin{array}{cc}
I & -h_{x}(\hat{x})^{T} \\
h_{x}(\hat{x}) & 0
\end{array}\right)\left(\begin{array}{c}
\dot{\hat{x}} \\
\dot{\lambda}
\end{array}\right)=\left(\begin{array}{c}
f(\hat{x}, u)+v(u, \hat{x}, \lambda, t) \\
\dot{y}
\end{array}\right) .
$$

But this DAE is in fact an ODE because, thanks to the assumption that the measurements are independent, $h_{x}(\hat{x})$ has full row rank which implies that the matrix on the left hand side of $(2.7)$ is invertible.

For (2.4)-(2.5) to be a proper relaxation of (1.1)-(1.2), by setting $\lambda=0$ in (2.4)-(2.5), we should get the equations in (1.1)-(1.2). This clearly implies that for $\lambda=0$, we must have

$$
v(u, \hat{x}, \lambda, t)=0 .
$$

In this report, we shall consider the case where $v$ can be expressed as

$$
v(u, \hat{x}, \lambda, t)=\Gamma(\hat{x}, u) \lambda .
$$

This of course is not the most general formulation. It is however enough for the approach presented in this report. So here we shall consider the canonical DAE observer:

$$
\begin{aligned}
\dot{\hat{x}} & =f(\hat{x}, u)+h_{x}(\hat{x})^{T} \dot{\lambda}+\Gamma(\hat{x}, u) \lambda \\
0 & =y-h(\hat{x}) .
\end{aligned}
$$

We have shown than (2.10)-(2.11) is a proper relaxation and has index 1. But this is not enough, we must show that $\lambda$ and $\lambda$ actually converge to zero (which under observability assumption implies that $\hat{x}$ converges to the unknown state $x$ ) for proper choice of $\Gamma$. Let us examine the convergence issue on an example.

Example 2.1: Consider the following system

$$
\left\{\begin{array}{l}
\dot{x}_{1}=\sin \left(x_{2}\right) \\
\dot{x}_{2}=-x_{2}+x_{1} u \quad, y=x_{2}
\end{array}\right.
$$

$\operatorname{RR} \mathbf{n}^{\circ} 2677$ 
where input $u$ is uniformly continuous and bounded. The observer for this system, as we have proposed in (2.10)-(2.11), is the DAE:

$$
\begin{aligned}
\dot{\hat{x}}_{1} & =\sin \left(\hat{x}_{2}\right)+\gamma_{1} \lambda \\
\dot{\hat{x}}_{2} & =-\hat{x}_{2}+\hat{x}_{1} u+\dot{\lambda}+\gamma_{2} \lambda \\
0 & =y-\hat{x}_{2}
\end{aligned}
$$

where $\gamma_{i}$ denotes the $i$-th entry of $\Gamma$. It is straightforward to verify that this DAE has index 1 and that it can easily be integrated using standard DAE solvers. Let us now examine the "error equation". Let

$$
\tilde{x}=\hat{x}-x
$$

Then from (2.12) and (2.13)-(2.15), besides $\tilde{x}_{2}=0$, we obtain:

$$
\begin{aligned}
\dot{\tilde{x}}_{1} & =\gamma_{1} \lambda \\
0 & =\tilde{x}_{1} u+\dot{\lambda}+\gamma_{2} \lambda
\end{aligned}
$$

which can be expressed as the following ODE:

$$
\left(\begin{array}{c}
\dot{\tilde{x}}_{1} \\
\dot{\lambda}
\end{array}\right)=\left(\begin{array}{cc}
0 & \gamma_{1} \\
-u & -\gamma_{2}
\end{array}\right)\left(\begin{array}{c}
\tilde{x}_{1} \\
\lambda
\end{array}\right)
$$

So the error equation is a linear equation but depends on the known (measured) input $u$. In this case, we can find $\Gamma$ by a simple Lyapunov argument. In particular, consider the Lyapunov function

$$
V=\tilde{x}_{1}^{2}+\alpha \lambda^{2}, \quad \alpha>0 .
$$

Then,

$$
\dot{V}=2\left(\gamma_{1}-\alpha u\right) \tilde{x}_{1} \lambda-2 \gamma_{2} \lambda^{2}
$$

Let

$$
\gamma_{1}=\alpha u, \quad \gamma_{2}>0,
$$

then $V$ is decreasing and $\lambda$ converges to zero (Theorem 4.8 of [10]). This, using the fact that $\dot{\lambda}$ is uniformly continuous, implies that $\dot{\lambda}$ converges to zero as well. Thus $\lambda$ is a proper relaxation.

Note that just because the relaxation is proper, it does not mean that $\hat{x}_{1}$ converges to $x_{1}$. For example, if $u$ is constant and equal to $0, x_{1}$ is completely decoupled from $y$ and clearly cannot be estimated. It is straightforward to verify that in this case the original system is not observable. To guarantee observability, we need to make additional assumptions on $u$. In particular, if $u$ is "persistently exciting", as $\lambda$ and $\dot{\lambda}$ go to zero, thanks to (2.18), $\tilde{x}_{1}$ should go to zero as well.

In this example, the error equation was linear for the canonical DAE observer; it would not be linear for standard ODE observers. This of course is not always the case, however, 
since in the DAE approach, we can substitute the measured states by the corresponding outputs which are common to both the original system and the observer, we often obtain a simpler, "more linear" error equation, compared to the original system or what we would obtain using standard observers. This is an important advantage of the DAE approach which is clearly illustrated by the following example.

Example 2.2: Consider the system

$$
\begin{aligned}
& \dot{x}=A x+g(x) \\
& y=C x
\end{aligned}
$$

where $A$ and $C$ are constant matrices and $g$ is such that there exists a function $j$ satisfying

$$
g(x)=j(C x)
$$

for all $x$. The canonical DAE observer in this case is:

$$
\begin{aligned}
& \dot{\hat{x}}=A \hat{x}+g(\hat{x})+C^{T} \dot{\lambda}+\Gamma \lambda \\
& y=C \hat{x} .
\end{aligned}
$$

But thanks to $(2.25), g(\hat{x})=g(x)$, so the error equation becomes

$$
\begin{aligned}
& \dot{\tilde{x}}=A \tilde{x}+C^{T} \dot{\lambda}+\Gamma \lambda \\
& y=C \tilde{x}
\end{aligned}
$$

which is linear. This is reminiscent of nonlinear observer design and in particular the construction of the observer normal form. Note however that in the DAE approach, the output injection which linearizes the error dynamics is done automatically because it is built in the formulation of the observer.

\subsection{Stability Analysis}

In the general case, we cannot express the estimation error in terms of a linear system. So, we consider here the local behavior of the error around zero. To carry out the local analysis, we need to make some assumptions.

Assumptions A: Initial condition $x(0)$ and input function $u(t)$ are such that if we let $x(t)$ denote the solution of (1.1)-(1.2), we have the following properties:

- $f$, and its first and second order partial derivatives with respect to $x$ are bounded in a neighborhood of $(x(t), u(t))$, uniformly in $t$,

- $h_{x}$ and its partial derivative with respect to $x$ are bounded in a neighborhood of $x(t)$, uniformly in $t$, 
- $\left(h_{x} h_{x}^{T}\right)^{-1}$ and $\Gamma$ are bounded in a neighborhood of $(x(t), u(t))$, uniformly in $t$.

Lemma 2.2 Under Assumptions $A$, if the estimation error, $\tilde{x}=\hat{x}-x$, associated with the canonical DAE observer (2.10)-(2.11) is sufficiently small, we have:

$$
\left(\begin{array}{cc}
I & -h_{x}(\hat{x})^{T} \\
0 & 0
\end{array}\right)\left(\begin{array}{c}
\dot{\tilde{x}} \\
\dot{\lambda}
\end{array}\right)=\left(\begin{array}{cc}
f_{x}(\hat{x}, u) & \Gamma(\hat{x}, u) \\
h_{x}(\hat{x}) & 0
\end{array}\right)\left(\begin{array}{c}
\tilde{x} \\
\lambda
\end{array}\right)+O\left(\|\tilde{x}\|^{2}\right) .
$$

Proof: Using (1.1)-(1.2) and (2.10)-(2.11), we obtain

$$
\begin{aligned}
\dot{\hat{x}}-\dot{x} & =f(\hat{x}, u)-f(x, u)+h_{x}(\hat{x})^{T} \dot{\lambda}+\Gamma(\hat{x}, u) \lambda \\
0 & =h(\hat{x})-h(x),
\end{aligned}
$$

from which, using

$$
\begin{aligned}
h(\hat{x})-h(x) & =h_{x}(\hat{x}) \tilde{x}+O\left(\|\tilde{x}\|^{2}\right) \\
f(\hat{x}, u)-f(x, u) & =f_{x}(\hat{x}, u) \tilde{x}+O\left(\|\tilde{x}\|^{2}\right)
\end{aligned}
$$

we get $(2.30)$.

In general, local behavior and in particular local stability of nonlinear DAE's cannot be studied by their linearizations. There exist however special cases where local stability can de deduced from stability of the linearization (for an example see [16]). It turns out that (2.30) corresponds to such a special case and that the linear time-varying DAE

$$
\left(\begin{array}{cc}
I & -h_{x}(\hat{x})^{T} \\
0 & 0
\end{array}\right) \dot{\xi}=\left(\begin{array}{cc}
f_{x}(\hat{x}, u) & \Gamma(\hat{x}, u) \\
h_{x}(\hat{x}) & 0
\end{array}\right) \xi
$$

can be used to establish local stability property of the nonlinear error equation associated with the canonical DAE observer.

Theorem 2.1 Under Assumptions A, if the origin in (2.35) is exponentially stable, the estimation error $\tilde{x}=\hat{x}-x$, associated with the canonical DAE observer, converges exponentially to zero provided $\hat{x}(0)-x(0)$, and $\lambda(0)$ are sufficiently small.

Proof: Consider the coordinate transformation:

$$
Q(t)\left(\begin{array}{l}
\xi_{1} \\
\xi_{2}
\end{array}\right)=\left(\begin{array}{c}
\dot{\tilde{x}} \\
\dot{\lambda}
\end{array}\right)
$$

where

$$
\left(\begin{array}{ll}
Q_{11}(t) & Q_{12}(t) \\
Q_{21}(t) & Q_{22}(t)
\end{array}\right)=\left(\begin{array}{cc}
I-h_{x}(\hat{x}(t))^{T}\left(h_{x}(\hat{x}(t)) h_{x}(\hat{x}(t))^{T}\right)^{-1} h_{x}(\hat{x}(t)) & h_{x}(\hat{x}(t))^{T} \\
-\left(h_{x}(\hat{x}(t)) h_{x}(\hat{x}(t))^{T}\right)^{-1} h_{x}(\hat{x}(t)) & I
\end{array}\right) .
$$


For $\tilde{x}$ sufficiently small, Assumptions A hold also for the time trajectory $(\hat{x}, u(t))$ in which case it is straightforward to show that $Q(t)$ is bounded and has a bounded inverse. Expressing (2.30) in this new coordinate system, we obtain (dropping the explicit dependence in $t$ to simplify the notations), assuming that $\tilde{x}$ and $\lambda$ are sufficiently small,

$$
\begin{aligned}
\dot{\xi}_{1}= & \left(f_{x}(\hat{x}, u) Q_{11}+\Gamma(\hat{x}, u) Q_{21}-\dot{Q}_{11}+h_{x}(\hat{x})^{T} \dot{Q}_{21}\right) \xi_{1}+ \\
& \left(f_{x}(\hat{x}, u) Q_{12}+\Gamma(\hat{x}, u) Q_{22}-\dot{Q}_{12}+h_{x}(\hat{x})^{T} \dot{Q}_{22}\right) \xi_{2}+O\left(\left\|\begin{array}{l}
\xi_{1} \\
\xi_{2}
\end{array}\right\|^{2}\right) \\
0= & \left(h_{x}(\hat{x}) Q_{12}-\dot{Q}_{22}\right) \xi_{2}+O\left(\left\|\begin{array}{c}
\xi_{1} \\
\xi_{2}
\end{array}\right\|^{2}\right) .
\end{aligned}
$$

But,

$$
\begin{aligned}
Q_{12} & =h_{x}(\hat{x})^{T} \\
Q_{22} & =I \\
\dot{Q}_{22} & =0 \\
h_{x}(\hat{x})^{T} \dot{Q}_{21} & =\dot{Q}_{11}-\dot{h}_{x}(\hat{x})^{T} Q_{21}
\end{aligned}
$$

which can be used to simplify (2.38)-(2.39) as follows:

$$
\begin{aligned}
\dot{\xi}_{1} & =A(t) \xi_{1}+B(t) \xi_{2}+O\left(\left\|\begin{array}{l}
\xi_{1} \\
\xi_{2}
\end{array}\right\|^{2}\right) \\
0 & =\left(h_{x}(\hat{x}) h_{x}(\hat{x})^{T}\right) \xi_{2}+O\left(\left\|\begin{array}{c}
\xi_{1} \\
\xi_{2}
\end{array}\right\|^{2}\right)
\end{aligned}
$$

where

$$
\begin{aligned}
& A(t)=f_{x}(\hat{x}, u) Q_{11}+\Gamma(\hat{x}, u) Q_{21}-\dot{h}_{x}(\hat{x})^{T} Q_{21} \\
& B(t)=f_{x}(\hat{x}, u) Q_{12}+\Gamma(\hat{x}, u) Q_{22}-\dot{h}_{x}(\hat{x})^{T} .
\end{aligned}
$$

Since $h_{x}(\hat{x}) h_{x}(\hat{x})^{T}$ has a bounded inverse, from (2.45), we conclude that

$$
\xi_{2}=O\left(\left\|\xi_{1}\right\|^{2}\right) .
$$

On the other hand, $h_{x}(\hat{x})^{T}$ is bounded since

$$
\dot{h}_{x}(\hat{x})^{T}=\left.\frac{\partial h_{x}(x) \dot{\hat{x}}}{\partial x}\right|_{x=\hat{x}} ^{T}
$$

and using (2.7) and (1.2), we can show that

$$
\dot{\hat{x}}=f(\hat{x}, u)+O\left(\left\|\begin{array}{c}
\tilde{x} \\
\lambda
\end{array}\right\|\right) .
$$

$\operatorname{RR} \mathbf{n}^{\circ} 2677$ 
So $A(t)$ and $B(t)$ are bounded. $B(t)$ bounded implies that $(2.44)-(2.45)$ is equivalent to

$$
\dot{\xi}_{1}=A(t) \xi_{1}+O\left(\left\|\xi_{1}\right\|^{2}\right) .
$$

And $A(t)$ bounded implies that (2.51) is locally exponentially stable if the linear time-varying system

$$
\dot{\xi}_{1}=A(t) \xi_{1}
$$

is exponentially stable (see for example [10], ch. 4). But exponential stability of (2.52) is equivalent to that of

$$
\begin{aligned}
\dot{\xi}_{1} & =A(t) \xi_{1}+B(t) \xi_{2} \\
0 & =\left(h_{x}(\hat{x}) h_{x}(\hat{x})^{T}\right) \xi_{2}
\end{aligned}
$$

which is nothing but (2.35) expressed in coordinate system

$$
\left(\begin{array}{l}
\xi_{1} \\
\xi_{2}
\end{array}\right)=Q(t)^{-1} \xi
$$

This proves the theorem.

Corollary 2.1 Suppose Assumptions A hold and the origin $\nu=0$ in

$$
\dot{\nu}=(F(\hat{x}(t), u(t))+\Gamma(\hat{x}(t), u(t)) H(\hat{x}(t))) \nu
$$

is exponentially stable where

$$
\begin{aligned}
H(\hat{x}) & =-\left(h_{x}(\hat{x}) h_{x}(\hat{x})^{T}\right)^{-1} h_{x}(\hat{x})^{T} \\
F(\hat{x}, u) & =f_{x}(\hat{x}, u)\left(I+\left(h_{x}(\hat{x})^{T}+\left.\frac{\partial h_{x}(x) f(\hat{x}, u)}{\partial x}\right|_{x=\hat{x}} ^{T}\right) H(\hat{x})\right) .
\end{aligned}
$$

Then, the estimation error $\hat{x}-x$, associated with the canonical DAE observer, converges exponentially to zero provided $\hat{x}(0)-x(0)$ and $\lambda(0)$ are sufficiently small.

Proof: If (2.56) is exponentially stable then

$$
\dot{\xi}_{1}=(F(\hat{x}(t), u(t))+\Gamma(\hat{x}(t), u(t)) H(\hat{x}(t))) \xi_{1}+O\left(\left\|\xi_{1}\right\|^{2}\right)
$$

is locally exponentially stable. But thanks to $(2.46)-(2.50),(2.59)$ is equivalent to $(2.51)$.

Let us suppose Assumptions A hold for all $\hat{x} \in \mathcal{X}$ and $u \in \mathcal{U}$. The observer design problem reduces to that of finding a $\Gamma$ that stabilizes (2.56). There is no systematic design procedure for constructing such a $\Gamma$. In some cases, a constant $\Gamma$ may suffice, and the problem becomes closely related to the robust stability problem with parametric uncertainty for which many solutions have been proposed in the $H_{\infty}$ control literature (see for example [7, 13]). In other cases, Lyapunov functions may be used for constructing more general $\Gamma$ 's. In Section 3, we will consider selection of $\Gamma$ to satisfy stability criteria related to extended-linearization considerations. Even though this method does not guarantee stability of (2.56), unless additional assumptions are made, it provides a systematic design procedure and yields a satisfactory observer in many situations, in particular when system inputs are slowly timevarying. 


\subsection{Relationship with reduced-order observers}

In this section, we examine the relationship between the DAE observer and reduce-order observers. In Example 2.1, we have seen that the part of the state which is directly observed, in this case $x_{2}$, is "estimated immediately" just as in case of reduced-order observers. The resemblance with reduced-order observers however ends here. In particular, observer (2.10)(2.11) does not have the well known problem of sensitivity to high frequency noise that reduced-order observers have. Let us illustrate this point by a simple example.

Example 2.3: In practice, there is no point applying the DAE observer to linear systems because, in this case, the DAE solver which is a linear descriptor system, can be transformed into an explicit linear system. However, examining the result on a simple example can shed some light on the properties of the observer and in particular its relationship with full and reduced-order observers. So, consider the linear system

$$
\left\{\begin{array}{l}
\dot{x}_{1}=x_{2} \\
\dot{x}_{2}=0
\end{array}, \quad y=x_{1}\right.
$$

The standard full-order observer for this problem (expressed in Laplace domain) is:

$$
\hat{x}=\frac{1}{s^{2}+\gamma_{1} s+\gamma_{2}}\left(\begin{array}{c}
\gamma_{1} s+\gamma_{2} \\
\gamma_{2} s
\end{array}\right) y
$$

where $\gamma_{1}$ and $\gamma_{2}$ can take any positive (to guarantee stability) values. The reduced-order observer (see [9], Section 4.3) for this system is:

$$
\hat{x}=\left(\begin{array}{c}
1 \\
\frac{\gamma s}{s+\gamma}
\end{array}\right) y
$$

where $\gamma$ is any positive scalar. To show why this reduced-order observer is more sensitive than the full order observer, let us suppose that the measurement available for the observer is not exactly $y(t)$ but rather $y(t)+\nu(t)$ where $\nu(t)$ is a small but high-frequency noise. It is easy to see that in the case of reduced-order observer, the estimation error is

$$
\tilde{x}=\left(\begin{array}{c}
1 \\
\frac{\gamma s}{s+\gamma}
\end{array}\right) \nu
$$

So the error in the estimation of $x_{1}$ is small $(=\nu(t))$ but the error in the estimation of $x_{2}$ can be very large since at high frequencies,

$$
\hat{x}_{2}=\frac{\gamma s}{s+\gamma} \nu \approx \gamma \nu
$$

and $\gamma$ must be large for fast convergence of the estimation error. The estimation error of $x_{2}$ in the full order case on the other hand is a low-pass filter applied to $\nu$ and it is less sensitive to high frequency noise.

$\operatorname{RR} \mathbf{n}^{\circ} 2677$ 
Let us now examine the observer obtained by DAE approaches. First consider the DAE (2.1), (2.3). If we let $\Gamma^{T}=\left[\begin{array}{ll}0 & \gamma\end{array}\right]$, regardless of the value of $\gamma$, we obtain the observer

$$
\hat{x}=\left(\begin{array}{l}
1 \\
s
\end{array}\right) y=\left(\begin{array}{c}
y \\
\dot{y}
\end{array}\right)
$$

which is the exact solution (in the absence of noise) but it is clearly very noise sensitive because the estimation error on $x_{2}$ equals $\dot{\nu}$. If we let $\Gamma^{T}=\left[\begin{array}{ll}1 & \gamma\end{array}\right]$, we obtain the reducedorder observer (2.62).

On the other hand, the canonical DAE observer (2.10)-(2.11) yields the following observer

$$
\hat{x}=\left(\begin{array}{c}
1 \\
\frac{\gamma_{2} s}{s^{2}+\gamma_{1} s+\gamma_{2}}
\end{array}\right) y
$$

which means that the estimation of $x_{1}\left(\hat{x}_{1}=y\right)$ is just as in the case of reduced-order observer but the estimation of $x_{2}$ (which was noise sensitive in the reduced-order case) is just as in the full order observer case.

\section{Extended-linearization method}

Nonlinear observers which are based on linearizing the observation error dynamics by transforming the system into observer normal form exist only for a restricted class of systems. Even if such a transformation does exist for a system, this method which requires the computation of multiple Lie derivatives involving $f$ and $h$ may not be appropriate if $f$ and $h$ do not model the plant very accurately. In some applications such as in robotics problems, the model is constructed from analytical considerations (physical laws) so even if the parameters of the model do not match exactly those of the real system, the derivatives of $f$ and $h$ are still meaningful. In other applications, however, such as those encountered in chemical and automotive industry, $f$ and $h$ are often identified and their derivatives can be very different from those of the real $f$ and $h$ (if they exist at all). Standard linearization around a single equilibrium point, in general, cannot be successfully applied to these systems either. Even though inputs of such systems are often reference signals which vary slowly or are constant over long periods of time, these systems usually have multiple and distant operating points. Extended linearization is one way to address these problems.

Extended linearization is a generalization of linearization around a single operating (equilibium) point. So let us first consider this latter case.

\subsection{Standard linearization}

Consider the stability of the equilibrium point $\left(u=u_{0}, x=\hat{x}=x_{0}, \lambda=0\right)$. In this case, thanks to Theorem 2.1, we need to study the stability of

$$
\left(\begin{array}{cc}
I & -h_{x}\left(x_{0}\right)^{T} \\
0 & 0
\end{array}\right) \dot{\xi}=\left(\begin{array}{cc}
f_{x}\left(x_{0}, u_{0}\right) & \Gamma \\
h_{x}\left(x_{0}\right) & 0
\end{array}\right) \xi
$$


Theorem 3.1 Suppose $h_{x}\left(x_{0}\right)$ has full row rank. Then, the finite eigen-modes of the DAE (3.1) correspond to the eigenvalues of the matrix $A_{0}-J C_{0}$ where $A_{0}=f_{x}\left(x_{0}, u_{0}\right), C_{0}=$ $h_{x}\left(x_{0}\right)$, and

$$
J=\left(A_{0} C_{0}^{T}+\Gamma\right)\left(C_{0} C_{0}^{T}\right)^{-1} .
$$

Proof: Finite eigen-modes of the DAE (3.1) are the finite eigen-modes of the matrix pencil

$$
\left\{\left(\begin{array}{cc}
I & -C_{0}^{T} \\
0 & 0
\end{array}\right),\left(\begin{array}{cc}
A_{0} & \Gamma \\
C_{0} & 0
\end{array}\right)\right\}
$$

which after a few simple elementary operations (which clearly does not affect the eigen-modes of the pencil) becomes

$$
\left\{\left(\begin{array}{ll}
I & 0 \\
0 & 0
\end{array}\right),\left(\begin{array}{cc}
A_{0}-J C_{0} & A_{0} C_{0}^{T}+\Gamma \\
0 & C_{0} C_{0}^{T}
\end{array}\right)\right\}
$$

where $J$ is given in (3.2). Note that $C_{0} C_{0}^{T}$ is invertible so the pencil has index 1 and

$$
\operatorname{det}\left(s\left(\begin{array}{cc}
I & 0 \\
0 & 0
\end{array}\right)-\left(\begin{array}{cc}
A_{0}-J C_{0} & A_{0} C_{0}^{T}+\Gamma \\
0 & C_{0} C_{0}^{T}
\end{array}\right)\right)=0
$$

if and only if

$$
\operatorname{det}\left(s I-\left(A_{0}-J C_{0}\right)\right)=0,
$$

proving the theorem.

Corollary 3.1 Let $A_{0}$ and $C_{0}$ be as defined in Theorem 3.1 and suppose that $C_{0}$ has full row rank and $\left(C_{0}, A_{0}\right)$ is observable. Then the finite eigen-modes of (3.1) can be arbitrarily assigned by proper choice of $\Gamma$.

Proof: Since $\left(C_{0}, A_{0}\right)$ is observable we can find $H$ such that the eigenvalues of $A_{0}-H C_{0}$ have the desired values. Now let

$$
\Gamma=\left(H C_{0}-A_{0}\right) C_{0}^{T}
$$

To show that this choice of $\Gamma$ does the job, we use the result of Theorem 3.1 from which we get that the finite eigen-modes of (3.1) equal the eigenvalues of

$$
\begin{aligned}
A_{0}-J C_{0} & =A_{0}-\left(A_{0} C_{0}^{T}+\Gamma\right)\left(C_{0} C_{0}^{T}\right)^{-1} C_{0} \\
& =A_{0}-H C_{0} .
\end{aligned}
$$

This completes the proof of the corollary.

So in the context of standard linearization, $\Gamma$ can be constructed as proposed in (3.5).

$\mathrm{RR} \mathbf{n}^{\circ} 2677$ 


\subsection{Extended linearization}

The idea of Extended-linearization in observer design has been introduced in $[2,3]$. The main idea in this approach, which is closely related to the gain scheduling technique, is to guarantee local stability of the error dynamics of the observer not just around an equilibrium point but for a family of equilibrium points.

Definition $3.1 \mathcal{E} \subset \mathcal{U} \times \mathcal{X}$ is called an equilibrium set if all $\left(u_{0}, x_{0}\right) \in \mathcal{E}$ satisfy $f\left(x_{0}, u_{0}\right)=$ 0 .

Theorem 3.2 Let $\mathcal{E}$ be an equilibrium set such that for all $\left(u_{0}, x_{0}\right) \in \mathcal{E}$, we have

1. $h_{x}\left(x_{0}\right)$ has full row rank;

2. $\left(h_{x}\left(x_{0}\right), f_{x}\left(x_{0}, u_{0}\right)\right)$ is observable.

Then there exists $\Gamma(\hat{x}, u)$ such that the error dynamics associated with observer (2.10)-(2.11) is locally stable around every equilibrium point in $\mathcal{E}$ provided $\tilde{x}(0)=\hat{x}(0)-x(0)$ is sufficiently small.

Proof: Let $J(\hat{x}, u)$ be an $n \times p$ matrix with entries which are functions of $\hat{x} \in \mathcal{X}$ and $u \in \mathcal{U}$ such that $f_{x}(\hat{x}, u)-J(\hat{x}, u) h_{x}(\hat{x})$ is Hurwitz for all $(u, \hat{x}) \in \mathcal{E}$ and let

$$
\Gamma(\hat{x}, u)=\left(J(\hat{x}, u) h_{x}(\hat{x})-f_{x}(\hat{x}, u)\right) h_{x}(\hat{x})^{T} .
$$

To show that this $\Gamma$ satisfies the conditions of Theorem 3.2, we first need to show the following key lemma.

Lemma 3.1 Let $\left(u_{0}, x_{0}\right) \in \mathcal{E}$ and consider an equilibrium point $x=x_{0}, \hat{x}=\hat{x}_{0}$, and $\lambda=\lambda_{0}$ for (1.1)-(1.2) and (2.10)-(2.11) associated with constant input $u_{0}$, i.e.,

$$
\begin{aligned}
0 & =f\left(x_{0}, u_{0}\right) \\
0 & =f\left(\hat{x}_{0}, u_{0}\right)+\Gamma\left(\hat{x}_{0}, u_{0}\right) \lambda_{0} \\
y & =h\left(x_{0}\right)=h\left(\hat{x}_{0}\right)
\end{aligned}
$$

where $\Gamma$ is defined in (3.7). Then there exists $\delta>0$ such that if $\left\|\hat{x}_{0}-x_{0}\right\|<\delta$, (3.8)-(3.10) imply that $\hat{x}_{0}=x_{0}$ and $\lambda_{0}=0$.

Proof: Suppose for all $\epsilon<\delta$ there exist $\hat{x}_{0}, x_{0}$ and $\lambda_{0}$ satisfying (3.8)-(3.10) such that $\left\|\hat{x}_{0}-x_{0}\right\|<\epsilon$, and $\tilde{x}_{0}=\hat{x}_{0}-x_{0}$ and/or $\lambda_{0}$ is not zero. For $\epsilon$ sufficiently small, from (3.8)-(3.10), with $O\left(\epsilon^{2}\right)$ accuracy, we get

$$
\left(\begin{array}{cc}
f_{x}\left(x_{0}, u_{0}\right) & \Gamma\left(x_{0}, u_{0}\right) \\
h_{x}\left(x_{0}\right) & 0
\end{array}\right)\left(\begin{array}{c}
\tilde{x}_{0} \\
\lambda_{0}
\end{array}\right)=0 .
$$


So to prove the Lemma we must show that the matrix in (3.11), which using (3.7) can be expressed as

$$
\left(\begin{array}{cc}
f_{x}\left(x_{0}, u_{0}\right) & \left(J\left(x_{0}, u\right) h_{x}\left(x_{0}\right)-f_{x}\left(x_{0}, u_{0}\right)\right) h_{x}\left(x_{0}\right)^{T} \\
h_{x}\left(x_{0}\right) & 0
\end{array}\right),
$$

is invertible. But it is easy to show that, by elementary operations, this matrix can be transformed into

$$
\left(\begin{array}{cc}
f_{x}\left(x_{0}, u_{0}\right)-J\left(x_{0}, u\right) h_{x}\left(x_{0}\right) & 0 \\
h_{x}\left(x_{0}\right) & h_{x}\left(x_{0}\right) h_{x}\left(x_{0}\right)^{T}
\end{array}\right),
$$

which is invertible because $(1,1)$-block entry is Hurwitz by construction and the $(2,2)$-block entry is invertible because $h_{x}\left(x_{0}\right)$ has full row rank. This proves the Lemma.

Thanks to Lemma 3.1, we know that the equilibrium points around which we need to linearize (1.1)-(1.2) and (2.10)-(2.11) coincide as far as $x$ is concerned and the equilibrium point $\lambda$ in (2.10)-(2.11) cannot be anything else but 0 . So we are in the same situation as in the standard linearization case discussed earlier, for every $\left(u_{0}, x_{0}\right) \in \mathcal{E}$. By construction $f_{x}\left(x_{0}, u_{0}\right)-J\left(x_{0}, u\right) h_{x}\left(x_{0}\right)$ is Hurwitz, so $\Gamma$ as defined in (3.7) locally stabilizes the system around all equilibrium points (see the proof of the corollary of Theorem 3.1).

There are various ways to construct matrix $J(\hat{x}, u)$ (and thus $\Gamma(\hat{x}, u)$ ). This matrix must be selected such that $f_{x}(\hat{x}, u)-J(\hat{x}, u) h_{x}(\hat{x})$ is Hurwitz for all $(u, \hat{x}) \in \mathcal{E}$. In some cases, it is straightforward to do that for all $(u, \hat{x})$. In other cases however, we need to restrict the domain to $\mathcal{E}$ to reduce complexity or avoid singularities. Let $A(\hat{x}, u)$ and $C(\hat{x}, u)$ denote respectively $f_{x}(\hat{x}, u)$ and $h_{x}(\hat{x})$ restricted to $\mathcal{E}$ :

$$
\begin{aligned}
A(\hat{x}, u) & =\left.f_{x}(\hat{x}, u)\right|_{(u, \hat{x}) \in \mathcal{E}} \\
C(\hat{x}, u) & =\left.h_{x}(\hat{x})\right|_{(u, \hat{x}) \in \mathcal{E}}
\end{aligned}
$$

Then, $J(\hat{x}, u)$ must be selected in such a way that $A(\hat{x}, u)-J(\hat{x}, u) C(\hat{x}, u)$ is Hurwitz. In following sections, we give two methods for constructing $J(\hat{x}, u)$.

Note that, in general, $A(\hat{x}, u)$ and $C(\hat{x}, u)$ are not unique and can be expressed in different ways. But, it is not always possible to express them independent of $\hat{x}$ (which means that equilibrium points cannot always be parameterized by inputs). To illustrate this point, let us consider the following simple example which has no input $u$.

$$
\left\{\begin{array}{l}
\dot{x}_{1}=x_{2} \\
\dot{x}_{2}=x_{1} x_{2}
\end{array}, \quad y=x_{1}\right.
$$

Clearly the equilibrium point corresponds to $x_{2}=0$ and $x_{1}$ arbitrary which implies that

$$
A(\hat{x}, u)=\left(\begin{array}{cc}
0 & 1 \\
0 & \hat{x}_{1}
\end{array}\right) \text {. }
$$

In this case $C(\hat{x}, u)=\left(\begin{array}{ll}1 & 0\end{array}\right)$. 


\subsection{Exact eigenvalue placement}

If $p=1$ (single output case), we can use Ackermann's formula [1] to construct $J(\hat{x}, u)$ such that

$$
\sigma_{i}(\hat{x}, u)=\text { eigenvalues of }[A(\hat{x}, u)-J(\hat{x}, u) C(\hat{x}, u)]
$$

have desired constant values. Let $\omega(s)$ be the polynomial having $\sigma_{i}$ 's for roots where $\sigma_{i}$ 's are the (constant) desired eigenvalues, then

$$
J(\hat{x}, u)=\omega(A(\hat{x}, u)) \mathcal{O}^{-1}\left(\begin{array}{c}
0 \\
0 \\
\vdots \\
1
\end{array}\right)
$$

where $\mathcal{O}$ denotes the observability matrix:

$$
\mathcal{O}=\left(\begin{array}{c}
C(\hat{x}, u) \\
C(\hat{x}, u) A(\hat{x}, u) \\
\vdots \\
C(\hat{x}, u) A(\hat{x}, u)^{n-1}
\end{array}\right) .
$$

The nice thing about this formula is that it gives an explicit expression for $J(\hat{x}, u)$. There is no equivalent formula for the $p>1$ (multi-output) case, since imposing $\omega$ does not uniquely determine $J$. However, if $A(\hat{x}, u)$ is cyclic for all admissible equilibrium points (of interest), we can find a $1 \times p$ vector $v(\hat{x}, u)$ such that the pair $(v(\hat{x}, u) C(\hat{x}, u), A(\hat{x}, u))$ is observable (in fact "almost any" vector $v$ does the job, and in particular we can take $v$ to be constant). Then using Ackermann's formula on this new pair we get:

$$
J_{1}(\hat{x}, u)=\omega(A(\hat{x}, u)) \mathcal{O}_{1}^{-1}\left(\begin{array}{c}
0 \\
0 \\
\vdots \\
1
\end{array}\right)
$$

where $\mathcal{O}_{1}$ denotes the observability matrix:

$$
\mathcal{O}_{1}=\left(\begin{array}{c}
v C(\hat{x}, u) \\
v C(\hat{x}, u) A(\hat{x}, u) \\
\vdots \\
v C(\hat{x}, u) A(\hat{x}, u)^{n-1}
\end{array}\right)
$$

and thus we can let

$$
J(\hat{x}, u)=J_{1}(\hat{x}, u) v
$$

If $A(\hat{x}, u)$ is not cyclic, it is possible to find a preliminary output injection $J_{p}$ such that

$$
A_{p}(\hat{x}, u)=A(\hat{x}, u)-J_{p} C(\hat{x}, u)
$$

is cyclic so that the previous method can be applied again (see [9], Section 7.1.2). 


\subsection{Regional eigenvalue placement}

Often, the exact locations of the eigenvalues of the error dynamics, $\sigma_{i}(\hat{x}, u)$, are not so important. In many cases, it is enough to require that they be "sufficiently stable", i.e.,

$$
\forall(\hat{x}, u), \quad \operatorname{Re}\left(\sigma_{i}(\hat{x}, u)\right)<-\alpha
$$

for some positive scalar $\alpha$.

Lemma 3.2 Let $X$ be any positive definite matrix and let

$$
P(\hat{x}, u)>0
$$

be the unique positive definite solution of the Riccati equation

$2 \alpha P(\hat{x}, u)+A(\hat{x}, u) P(\hat{x}, u)+P(\hat{x}, u) A(\hat{x}, u)^{T}-P(\hat{x}, u) C(\hat{x}, u)^{T} X C(\hat{x}, u) P(\hat{x}, u)+I=0$.

Then (3.23) holds if

$$
J(\hat{x}, u)=\frac{1}{2} P(\hat{x}, u) C(\hat{x}, u)^{T} X .
$$

Proof: From (3.25) and (3.26), we obtain

$$
\begin{aligned}
2 \alpha P(\hat{x}, u)+(A(\hat{x}, u)-J(\hat{x}, u) C(\hat{x}, u)) P(\hat{x}, u)+P(\hat{x}, u)(A(\hat{x}, u)-J(\hat{x}, u) C(\hat{x}, u))^{T}+I & = \\
(\alpha I+A(\hat{x}, u)-J(\hat{x}, u) C(\hat{x}, u)) P(\hat{x}, u)+P(\hat{x}, u)(\alpha I+A(\hat{x}, u)-J(\hat{x}, u) C(\hat{x}, u))^{T}+I & =0
\end{aligned}
$$

but this is nothing but a Lyapunov equation which implies that $\alpha I+A(\hat{x}, u)-J(\hat{x}, u) C(\hat{x}, u)$ is stable which is equivalent to condition (3.23).

To show that (3.25) has a unique positive solution, simply note that it can be rewritten as

$$
(\alpha I+A(\hat{x}, u)) P(\hat{x}, u)+P(\hat{x}, u)(\alpha I+A(\hat{x}, u))^{T}-P(\hat{x}, u) C(\hat{x}, u)^{T} X C(\hat{x}, u) P(\hat{x}, u)+I=0
$$

which is a standard Riccati equation which has a unique positive solution provided

$$
\begin{aligned}
(C(\hat{x}, u), \alpha I+A(\hat{x}, u)) & \text { is observable, } \\
(\alpha I+A(\hat{x}, u), I) & \text { is controllable. }
\end{aligned}
$$

The first condition holds because $(C(\hat{x}, u), A(\hat{x}, u))$ is observable and the second is trivial.

Note that matrix $X$ (which may also depend on $u$ and $\hat{x}$ ) is an additional tunning parameter which can be used to adjust the behavior of the error dynamics.

Lemma 3.2 provides a straightforward method for constructing $J(\hat{x}, u)$ and thus $\Gamma(\hat{x}, u)$. However, it does not provide an explicit solution for it. To construct $J(\hat{x}, u)$, we need to solve the Riccati equation (3.25). This may or may not be feasible in real-time. In most cases, we need to precompute $J$ for a reasonably dense set of $u$ 's and $\hat{x}$ 's and use, in real-time, interpolation or similar methods commonly used in gain scheduling.

$\operatorname{RR} \mathbf{n}^{\circ} 2677$ 


\section{Implementation issues}

Numerical implementation is an issue which is rarely considered in the observer design literature. The reason for that is that, in most cases, the final implementation is done using standard code for ODE's which are known to be very reliable. Thanks to its particular structure, the canonical DAE solver can also be implemented using existing reliable codes; but in this case, DAE solvers.

Implementing observer (2.10)-(2.11) is not much different from implementing a standard ODE based observer; we simply have to use a DAE solver instead of an ODE solver.

Most DAE solvers are based on the BDF (Backward Differentiation Formula) method introduced originally in [8]. Consider the DAE

$$
F(t, z, \dot{z})=0 .
$$

The first order BDF method consists of discretizing (4.1) as follows

$$
F\left(t_{k+1}, z_{k+1}, \frac{z_{k+1}-z_{k}}{t_{k+1}-t_{k}}\right) \text {. }
$$

At each time step, $z_{k+1}$ is computed in terms of $z_{k}$ by solving a set of nonlinear algebraic equations (usually by Newton's Method). ${ }^{1}$ This single step method can be generalized to a multistep method by expressing $\dot{z}$ at $t=t_{k+1}$ by the derivative of a polynomial that interpolates the computed solutions $z_{j}, j=k+1-n, \ldots, k+1$, evaluated at $t=t_{k+1}$. The multi-step BDF DAE solver is particularly well suited for solving uniform index 1 problems.

Definition 4.1 ([5], Sec. 3.2) The nonlinear DAE (4.1) is said to be uniform index 1 if the index of the matrix pencil $\{A, B\}$, where $A=F_{\dot{z}}(t, z, \dot{z})$ and $B=F_{z}(t, z, \dot{z})$, is one for all $(t, z, \dot{z})$ in a neighborhood of the graph of the solution of (4.1), and if

1. the partial derivatives of $A$ with respect to $t, z, \dot{z}$ exist and are bounded in a neighborhood of the solution,

2. the rank of $A$ is constant in a neighborhood of the solution.

Expressing the canonical DAE observer (2.10)-(2.11) as in (4.1) yields:

$$
F\left(t,\left(\begin{array}{c}
\hat{x} \\
\lambda
\end{array}\right),\left(\begin{array}{c}
\dot{\hat{x}} \\
\dot{\lambda}
\end{array}\right)\right)=\left[\begin{array}{c}
\dot{\hat{x}}-f(\hat{x}, u(t))-h_{x}(\hat{x})^{T} \dot{\lambda}-\Gamma(\hat{x}, u(t)) \lambda \\
y(t)-h(\hat{x})
\end{array}\right]=0 .
$$

Lemma 4.1 Under Assumptions A, for $\hat{x}$ sufficiently close to $x$, the DAE observer (2.10)(D.11) is uniform index 1.

The proof is straightforward and is omitted.

\footnotetext{
${ }^{1}$ This is in contrast with the case of ODE solvers where explicit Euler discretization leads to an explicit expression for $z_{k+1}$.
} 
Theorem 4.1 ([5], Sec. 3.2) Let (4.1) be a uniform index 1 DAE on an interval $I=$ $\left[t_{0}, t_{0}+T\right]$. Then the numerical solution of (4.1) by the $k$-step BDF with fixed stepsize $\epsilon$ for $k<7$ converges to $O\left(\epsilon^{k}\right)$ if the initial condition is correct to $O\left(\epsilon^{k}\right)$ accuracy and if the Newton iteration on each step is solved to $O\left(\epsilon^{k+1}\right)$ accuracy.

Finding a correct initial condition for (2.10)-(2.11) is rather easy; in particular, any $\hat{x}(0)$ satisfying $y(0)=h(\hat{x}(0)$ ) and any $\lambda(0)$ (in particular $\lambda(0)=0$ ) constitute a correct initial condition. As for the choice of stepsize $\epsilon$ and order $k$ of the integrator, they depend on a number of factors related to the performance of the measuring device (for measuring $u$ and $y$ ) and the computational power available. For example, it does not make sense to have $\epsilon^{k}$ smaller than the magnitude of the measurement noise.

DAE solvers usually require that Jacobian matrices $F_{z}$ and $F_{\dot{z}}$ be provided, if not, they need to be estimated which can slow down the integration and increase the risk of failure. Based on (4.3), these matrices can be easily computed:

$$
\begin{aligned}
F\left(\begin{array}{l}
\hat{x} \\
\lambda
\end{array}\right) & =\left(\begin{array}{cc}
-f_{x}(\hat{x}, u(t))-\frac{\partial\left(h_{x}(\hat{x})^{T} \dot{\lambda}+\Gamma(\hat{x}, u(t)) \lambda\right.}{\partial \hat{x}} & \Gamma(\hat{x}, u(t)) \\
-h_{x}(\hat{x})^{2} & 0
\end{array}\right) \\
F\left(\begin{array}{c}
\dot{\hat{x}} \\
\dot{\lambda}
\end{array}\right) & =\left(\begin{array}{cc}
I & -h_{x}(\hat{x})^{T} \\
0 & 0
\end{array}\right) .
\end{aligned}
$$

A number of multi-step BDF codes are now available and widely distributed, such as DASSL [14]. For a real-time application, DASSL may not be appropriate since it varies the step size and in a real application, usually the measurements of $u$ and $y$ are available by fixed step sampling. Moreover, it does a lot of error control, to "protect" the user, which is not necessary for real time implementation. For experimentation however, DASSL provides a nice platform. The following example has been implemented using DASSL.

\section{Example}

Consider the model of a three-phase current motor used in [4].

$$
\left(\begin{array}{c}
\dot{x}_{1} \\
\dot{x}_{2} \\
\dot{x}_{3}
\end{array}\right)=\left(\begin{array}{c}
x_{2} \\
B_{1}-A_{1} x_{2}-A_{2} x_{3} \sin \left(x_{1}\right)-\frac{1}{2} B_{2} \sin \left(2 x_{1}\right) \\
u(t)-D_{1} x_{3}+D_{2} \cos \left(x_{1}\right)
\end{array}\right) \quad, \quad y=\left(\begin{array}{c}
x_{1} \\
x_{2}
\end{array}\right) .
$$

We use exact pole placement as discussed in the previous section to place the poles of the error dynamics at $(-10,-10,-10)$. First we need to compute $A(\hat{x}, u)$ and $C(\hat{x}, u)$. Then we have to find $J(\hat{x}, u)$ such that the eigenvalues of $A(\hat{x}, u)-J(\hat{x}, u) C(\hat{x}, u)$ are all equal to -10 for equilibrium points $(u, \hat{x})$. It turns out that we can place the eigenvalues for all $(u, \hat{x})$ so we don't need to explicity compute the set of equilibrium states $\mathcal{E}$. So let $A(\hat{x}, u)=f_{x}(\hat{x}, u)$ 
and $C(\hat{x}, u)=h_{x}(\hat{x})$ :

$$
\begin{aligned}
A(\hat{x}, u) & =\left(\begin{array}{ccc}
0 & 1 & 0 \\
-A_{2} \hat{x}_{3} \cos \left(\hat{x}_{1}\right)-B_{2} \cos \left(2 \hat{x}_{1}\right) & -A_{1} & -A_{2} \sin \left(\hat{x}_{1}\right) \\
-D_{2} \sin \left(\hat{x}_{1}\right) & 0 & -D_{1}
\end{array}\right) \\
C(\hat{x}, u) & =\left(\begin{array}{lll}
1 & 0 & 0 \\
0 & 1 & 0
\end{array}\right) .
\end{aligned}
$$

This system has more than one output, so we must find a $v$ such that the pair $(v C(\hat{x}, u), A(\hat{x}, u))$ is observable. The obvious choice is $v=\left(\begin{array}{ll}1 & 0\end{array}\right)$. Letting

$$
\omega(s)=(s+10)^{3}
$$

from (3.19) and (3.20), after some straightforward algebra, we get

$$
J_{1}(\hat{x}, u)=\left(\begin{array}{c}
30-A_{1}-D_{1} \\
-A_{2} \hat{x}_{3} \cos \left(\hat{x}_{1}\right)-B_{2} \cos \left(2 \hat{x}_{1}\right)+300-30 A_{1}+A_{1}^{2}-30 D_{1}+A_{1} D_{1}+D_{1}^{2} \\
-D_{2} \sin \left(\hat{x}_{1}\right)-\left(D_{1}-10\right)^{3} /\left(A_{2} \sin \left(\hat{x}_{1}\right)\right)
\end{array}\right) .
$$

Now using (3.21), we obtain

$$
\begin{aligned}
\Gamma(\hat{x}, u) & =(J(\hat{x}, u) C(\hat{x}, u)-A(\hat{x}, u)) C(\hat{x}, u)^{T} \\
& =\left(\begin{array}{cc}
30-A_{1}-D_{1} & -1 \\
300-30 A_{1}+A_{1}^{2}-30 D_{1}+A_{1} D_{1}+D_{1}^{2} & A_{1} \\
-\left(D_{1}-10\right)^{3} /\left(A_{2} \sin \left(\hat{x}_{1}\right)\right) & 0
\end{array}\right) .
\end{aligned}
$$

So, we get the canonical DAE observer:

$$
\left\{\begin{array}{rlr}
\dot{\hat{x}}_{1}= & \hat{x}_{2}+\dot{\lambda}_{1}+\left(30-A_{1}-D_{1}\right) \lambda_{1}-\lambda_{2} \\
\dot{\hat{x}}_{2}= & B_{1}-A_{1} \hat{x}_{2}-A_{2} \hat{x}_{3} \sin \left(\hat{x}_{1}\right)-\frac{1}{2} B_{2} \sin \left(2 \hat{x}_{1}\right)+\dot{\lambda}_{2}+ \\
& \quad\left(300-30 A_{1}+A_{1}^{2}-30 D_{1}+A_{1} D_{1}+D_{1}^{2}\right) \lambda_{1}+A_{1} \lambda_{2} \\
\dot{\hat{x}}_{3}= & u(t)-D_{1} \hat{x}_{3}+D_{2} \cos \left(\hat{x}_{1}\right)-\lambda_{1}\left(D_{1}-10\right)^{3} /\left(A_{2} \sin \left(\hat{x}_{1}\right)\right) \\
y_{1}(t)= & \hat{x}_{1} \\
y_{2}(t)= & \hat{x}_{2}
\end{array}\right.
$$

Simulation results for the same values used in [4], i.e.,

$$
D 1=.3222, D 2=1.9, A 1=.2703, A 2=12.01, B 1=39.19, B 2=-48.04
$$

are given in Figures 1 through 4 . The input is $u=2(1+\sin (2 t))$.

\section{6 conclusion}

In this report, we have shown that there are advantages in formulating observers as DAE's which can be implemented using existing DAE solvers. A design method based on the extended-linearization approach is presented. 


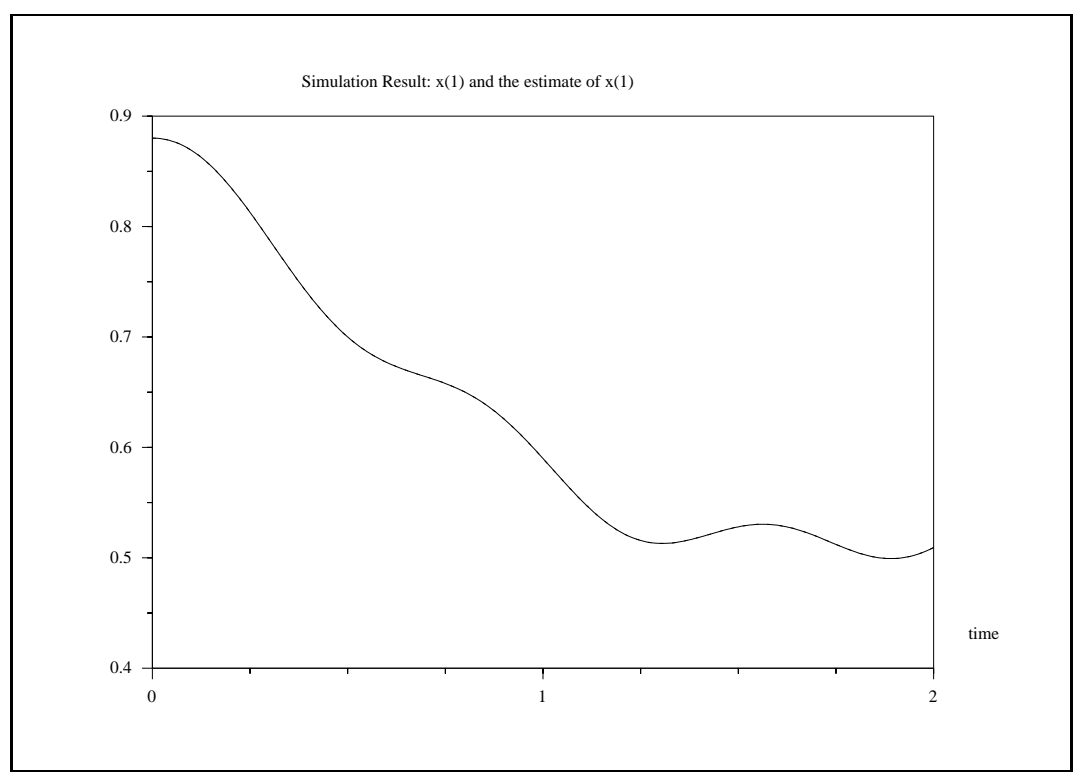

Figure 1: $x_{1}$ and $\hat{x}_{1}$ coincide exactly as expected 


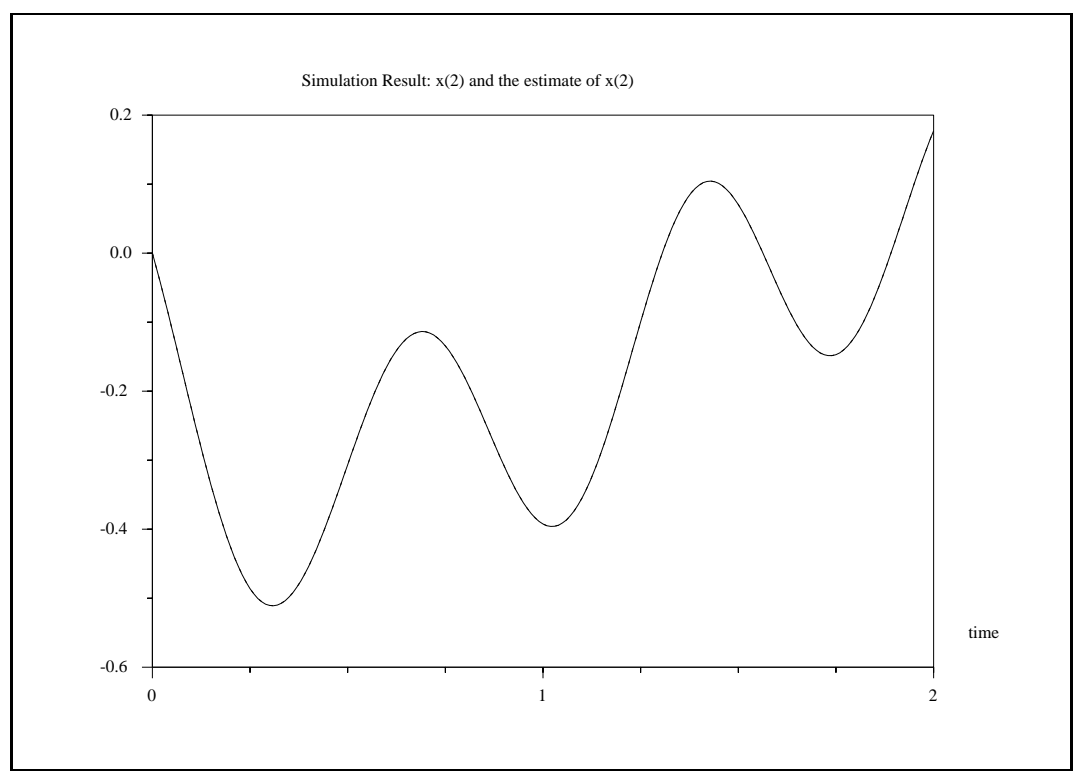

Figure 2: $x_{2}$ and $\hat{x}_{2}$ coincide exactly as expected 


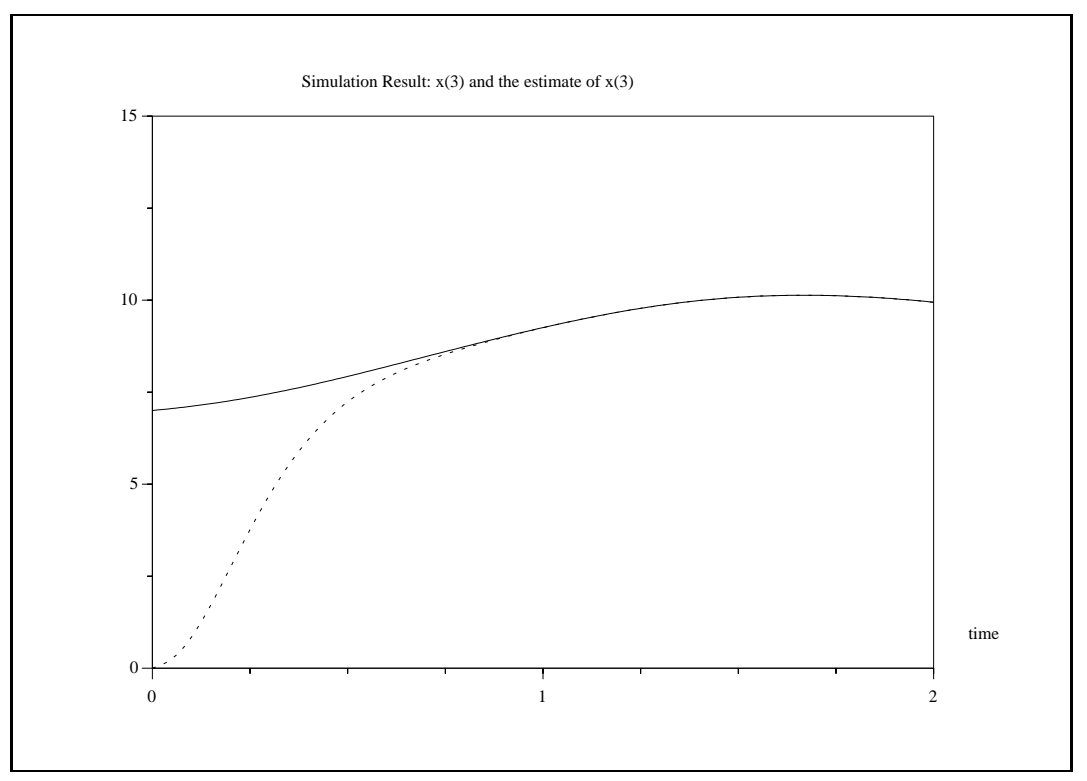

Figure 3: solid line: $x_{3}$, dashed line: $\hat{x}_{3}$ 


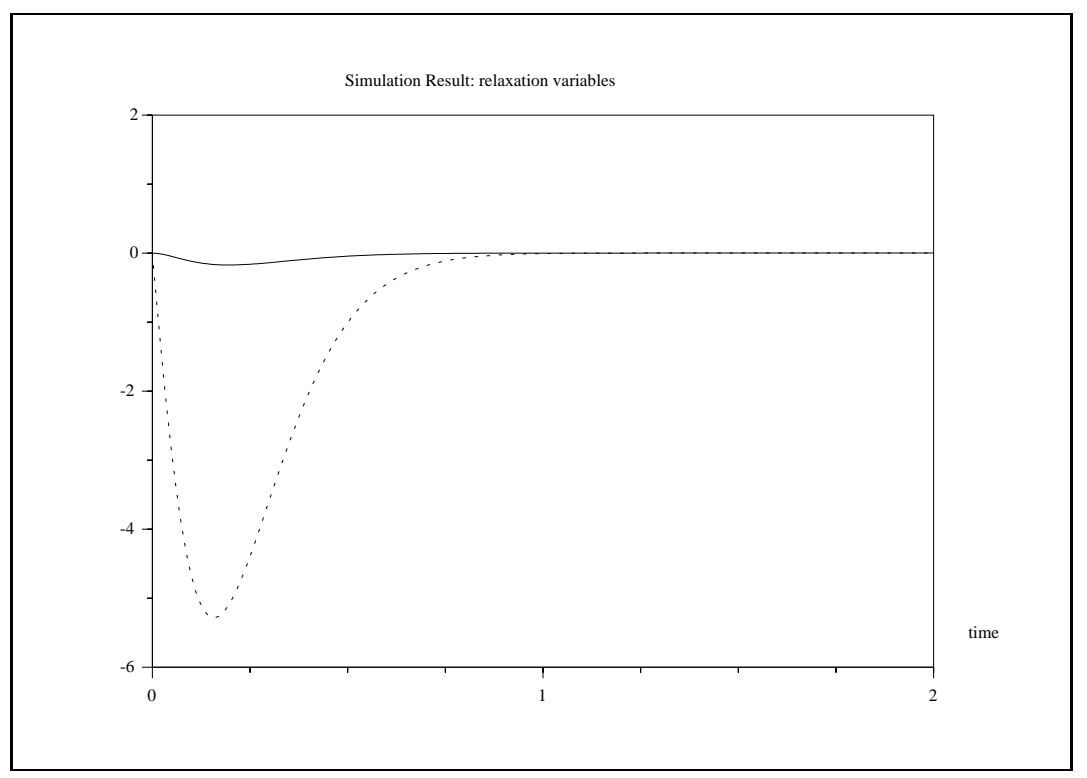

Figure 4: solid line: $\lambda_{1}$, dashed line: $\lambda_{2}$ 
There are many possible extensions to the work presented here. An immediate one is to allow the measurement equation depend on the input $u$, i.e., have $y=h(x, u)$ instead of (1.2) (this situation seldom comes up in real applications). All the results presented here can be easily extended to this case. A more interesting extension is to consider the observer problem with unknown inputs. Unknown inputs in a sense are relaxation variables, and the problem of estimation with unknown inputs falls nicely into the DAE framework. The difficulty in formulating the DAE observer in that case lies in proper combination of the unknown inputs and the (reduced) set of relaxation variables ( $\lambda$ 's) so that the resulting DAE has the required properties. The observer with unknown inputs has applications in residual generation for failure detection. In fact, the relaxation variable $\lambda$ can be directly interpreted as a residual, so that, in this case, the canonical DAE observer can be considered as a residual generator.

\section{References}

[1] Ackermann, J., Der Entwurf linearer Regelungssysteme in Zustandsraum, Regelungestechnik und Prozessedatenverarbeitung, vol. 7, 1972.

[2] Baumann, W. T. and Rugh, W. J., Feedback control of nonlinear systems by extended linearization, IEEE Trans. on Automat. Contr., vol. 31, no. 1, 1986.

[3] Baumann, W. T., Feedback control of multiinput nonlinear systems by extended linearization, IEEE Trans. on Automat. Contr., vol. 33, no. 2, 1988.

[4] Birk, J. and Zeitz, M., Extended Luenberger observer for non-linear multivariable systems, Int. J. Contr., vol. 47, no. 6, 1988.

[5] Brenan, K. E., Campbell, S. L., and Petzold, L. R., Numerical Solution of Initial-Value Problems in Differential-Algebraic Equations, North-Holland, 1989.

[6] Cobb, J. D., A unified theory of full-order and low-order observers based on singular system theory, IEEE Trans. on Automat. Contr., vol. 39, no. 12, 1994.

[7] Fan, M. K. H., Tits, A. L., and Doyle, J. C., Robustness in the presence of mixed parametric uncertainty and unmodeled dynamics, IEEE Trans. on Automat. Contr., vol. 36, no. 1, 1991.

[8] Gear, C. W., The simultaneous numerical solution of differential-algebraic equations, IEEE Trans. on Circuit Theory, vol. 18, 1971.

[9] Kailath, T., Linear Systems, Prentice-Hall, 1980.

[10] Khalil, H. K., Nonlinear Systems, Macmillan, 1992.

$\mathrm{RR} \mathrm{n}^{\circ} 2677$ 
[11] Michalska, H. and Mayne, D. Q., Moving horizon observers and observer-based control, IEEE Trans. on Automat. Contr., vol. 40, no. 6, 1995.

[12] Moraal, P. E. and Grizzle, J. W., Observer design for nonlinear systems with discretetime measurements, IEEE Trans. on Automat. Contr., vol. 40, no. 3, 1995.

[13] Petersen, I. R. and McFarlane, D. C., Optimal guaranteed cost control and filtering for uncertain linear systems, IEEE Trans. on Automat. Contr., vol. 39, no. 9, 1994.

[14] Petzold, L. R., A description of DASSL: a differential/algebraic system solver, in Scientific Computing, eds. R. S. Stepleman et al., North-Holland, 1983.

[15] Walcott, B. L., Corless, M. J., and Zak, S. H., Comparative study of non-linear stateobservation techniques, Int. J. Contr., vol. 45, no. 6, 1987.

[16] McClamroch, N. H. and Wang, D., Feedback stabilization and tracking of constrained robots, IEEE Trans. on Automat. Contr., vol. 33, no. 5, 1988. 


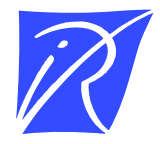

Unité de recherche INRIA Lorraine, Technopôle de Nancy-Brabois, Campus scientifique,

615 rue du Jardin Botanique, BP 101, 54600 VILLERS LÈS NANCY

Unité de recherche INRIA Rennes, Irisa, Campus universitaire de Beaulieu, 35042 RENNES Cedex

Unité de recherche INRIA Rhône-Alpes, 46 avenue Félix Viallet, 38031 GRENOBLE Cedex 1

Unité de recherche INRIA Rocquencourt, Domaine de Voluceau, Rocquencourt, BP 105, 78153 LE CHESNAY Cedex

Unité de recherche INRIA Sophia-Antipolis, 2004 route des Lucioles, BP 93, 06902 SOPHIA-ANTIPOLIS Cedex

Éditeur

INRIA, Domaine de Voluceau, Rocquencourt, BP 105, 78153 LE CHESNAY Cedex (France)

ISSN 0249-6399 